\title{
Machine learning in orthodontics: Challenges and perspectives
}

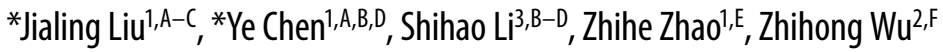 \\ 1 State Key Laboratory of Oral Diseases \& National Clinical Research Center for Oral Diseases, Department of Orthodontics, West China Hospital of Stomatology, \\ Sichuan University, Chengdu, China \\ ${ }^{2}$ College of Computer Science, Sichuan University, Chengdu, China \\ ${ }^{3}$ National Key Laboratory of Fundamental Science on Synthetic Vision, College of Computer Science, Sichuan University, Chengdu, China \\ A - research concept and design; B - collection and/or assembly of data; $C$ - data analysis and interpretation; \\ $D$ - writing the article; $E$ - critical revision of the article; $F$ - final approval of the article
}

Advances in Clinical and Experimental Medicine, ISSN 1899-5276 (print), ISSN 2451-2680 (online)

Adv Clin Exp Med. 2021;30(10):1065-1074

\section{Address for correspondence \\ Zhihong Wu}

E-mail:wuzhihong@scu.edu.cn

\section{Funding sources}

None declared

\section{Conflict of interest}

None declared

\section{Acknowledgements}

We acknowledge Dr. Wen Liao for his valuable suggestions.

*Jialing Liu and Ye Chen contributed equally to this work.

Received on March 26, 2021

Reviewed on June 3,2021

Accepted on June 9, 2021

Published online on 0ctober 5, 2021

\begin{abstract}
Artificial intelligence (Al) applications have significantly improved our everyday quality of life. The last decade has witnessed the emergence of up-and-coming applications in the field of dentistry. It is hopeful that Al, especially machine learning (ML), due to its powerful capacity for image processing and decision support systems, will find extensive application in orthodontics in the future. We performed a comprehensive literature review of the latest studies on the application of ML in orthodontic procedures, including diagnosis, decision-making and treatment. Machine learning models have been found to perform similar to or with even higher accuracy than humans in landmark identification, skeletal classification, bone age prediction, and tooth segmentation. Meanwhile, compared to human experts, ML algorithms allow for high agreement and stability in orthodontic decision-making procedures and treatment effect evaluation. However, current research on ML raises important questions regarding its interpretability and dataset sample reliability. Therefore, more collaboration between orthodontic professionals and technicians is urged to achieve a positive symbiosis between Al and the clinic.
\end{abstract}

Key words: neural network, orthodontics, machine learning, artificial intelligence (Al), convolutional neural network (CNN)

Cite as

Liu J, Chen Y, Li S, Zhao Z, Wu Z. Machine learning

in orthodontics: Challenges and perspectives.

Adv Clin Exp Med. 2021;30(10):1065-1074.

doi:10.17219/acem/138702

DOI

10.17219/acem/138702

Copyright

Copyright by Author(s)

This is an article distributed under the terms of the

Creative Commons Attribution 3.0 Unported (CC BY 3.0)

(https://creativecommons.org/licenses/by/3.0/) 


\section{Introduction}

The goal of orthodontic treatment is to restore individual normal occlusion and improve facial attractiveness in patients with malocclusion. Malocclusion is a common disease with high prevalence (up to $56 \%$ in the world). ${ }^{1}$ The diagnosis of malocclusion is made with an accurate measurement of distance, planes and angles according to landmarks of soft and hard tissues using lateral cephalogram and cone-beam computed tomography $(\mathrm{CBCT})$. Due to high spatial resolution and multi-direction presentation, $\mathrm{CBCT}$ can provide more accurate craniometric outcomes than lateral cephalograms. ${ }^{2}$ However, the definitions of the anatomic landmarks differ among orthodontists, so the outcomes of landmark coordinates and geometrical parameters vary significantly in either method and depend largely on the orthodontists' experience and the image quality. Moreover, the procedures of malocclusion diagnosis are time-consuming.

Treatment decision analysis plays a pivotal role in orthodontic procedures. For instance, orthodontists are often confronted with the choice between extraction and non-extraction orthodontic treatment. Multiple factors including tooth health, arch width and smile esthetics need to be taken into consideration to achieve an optimal clinical effect. ${ }^{3}$ Additionally, due to higher risk, the choice of orthodontic-orthognathic combined treatment calls for caution, especially in patients with severe skeletal malocclusion and asymmetric jaw deformity. ${ }^{4}$ Overall, orthodontic decision-making greatly influences the long-term prognosis, but, due to a lack of consensus on complicated cases, plans can vary among different orthodontists and even different cases treated by the same orthodontists. Thus, effective methods are needed to help human experts improve their treatment planning and reduce the inter-physician variability. Moreover, a standard therapy system could provide clinical instructions for young doctors, particularly as the popularity of personalized orthodontics increases and the need for customized plans increases the complexity of treatment decisions.

With the development of computer-aided design/ computer-aided manufacturing (CAD/CAM), thermoformed tooth aligners have become a popular choice for adult patients with malocclusion. ${ }^{5}$ Tooth segmentation from intraoral scanning or CBCT is a pivotal step for computer-aided aligner design. ${ }^{6}$ Although some mathematical methods, including region-based and feature curve-based segmentation, have been studied in tooth segmentation, the digital image results were not ideal, due to the distorted display of interdental and tooth-gingiva areas as well as curvature noises, which affect the manufacturing accuracy. ${ }^{7}$ Therefore, tooth segmentation based on artificial intelligence (AI) has become a promising method to acquire high precision.

\section{Objectives}

Personalized orthodontic treatment poses challenges for orthodontists in diagnosis, decision-making and treatment. Fortunately, considerable increases in the performance of integrated circuits have allowed AI to contribute substantially to handling images and decision-making due to developments in facial recognition and expert systems. Thus far, AI has performed impressively in many applications in dentistry, especially in periodontology, prosthodontics and endodontics, and the research on AIbased orthodontic treatment is burgeoning. ${ }^{8}$ In this paper, we present current applications of AI in the field of orthodontics and we attempt to predict some future applications.

\section{Artificial intelligence}

\section{The introduction of Al}

At the beginning of the 1980s, a computer-aided tool called an "expert system" based on rules was strongly promoted by the Japanese government. ${ }^{9}$ However, due to an inefficient search and indefinite weight relationships between rules, the system could not meet the actual tasks. The emergence of machine learning (ML) solved the dilemma; it showed improved predictive performance by extracting internal connections among manually input features; such process is known as handcraft feature engineering.10 In the next stage of development, automatic feature engineering based on deep learning technology could replace the process of manual extraction. ${ }^{11}$ As a branch of ML, deep learning relies on neural networks and it achieved the breakthrough of processing high-dimensional structure data, such as images.

Artificial neural networks are based on a collection of connected units or nodes called artificial neurons, which can mimic the neurons in the biological brain. Each connection is like a synapse in a biological brain, which can transmit signals to neighboring neurons. The signal may propagate from input layer to output layer via several hidden layers. Every layer consists of many neurons which are connected to the neurons of previous layers. The synapses represent weighted parameter (w) and constant (b) values. Deep learning refers to optimizing the weighted parameters automatically by minimizing the errors between output and label input. ${ }^{12}$ The reduction of loss functions of mini-batch using gradient descent, called stochastic gradient descent (SGD), has been widely applied to train neural networks. ${ }^{13}$

As an evolution of deep learning, convolutional neural network (CNN) enhanced local feature extraction and reduced sensitivity to changes in the position and size of images. In CNN, the hidden layers were replaced by convolutional layers, pooling layers and fully connected layers. ${ }^{14}$ In convolutional layers, convolutional kernels with 
the entire depth of the input images are convoluted with the feature values of source pixels, and the calculated outcomes are projected to destined pixels. ${ }^{15}$ The kernels slide over the feature maps at certain places, and the parameters of each kernel could be shared within every layer. ${ }^{16}$ Pooling layers can narrow input volumes and decrease calculation amounts. Common pooling functions include average pooling and max pooling. Fully connected layers are assigned before output layers, and the neurons are connected with all the neurons of preceding layers. ${ }^{17}$

\section{Four Al-driven tasks in dentistry}

The 4 major AI-driven tasks in dentistry are classification, regression, detection, and segmentation. The most well-known task in the AI field is classification. This task means assigning objects or features to pre-specified categories. ${ }^{18}$ Classification models have been widely studied in diagnosis of oral diseases, such as periodontitis and caries. ${ }^{10}$ The classification neural network will extract features in hidden layers and map optimal categories according to the likelihood in output layers. ${ }^{19}$ The common classification algorithms include naïve Bayesian, support vector machine (SVM), decision tree, and artificial neural network (ANN). ${ }^{20}$ Due to convolution and pooling, CNN can handle high-dimensional data such as high-resolution images. ${ }^{21}$ For instance, $\mathrm{CNN}$ has shown its potential in oral cancer pathologic diagnosis through its handling of shape, color and texture features of nuclei. ${ }^{22}$

In applications based on AI, the regression analysis is used to estimate the relationships between multiple variables and predict dependent variables based on independent variables. ${ }^{23}$ In dentistry, researchers transformed the procedure of predicting numerical results into a procedure for determinations. For example, clinical decision support systems based on regression can estimate color change after tooth whitening. ${ }^{8}$ Additionally, regression methods can be integrated into ML models based on SVM and neural network. The accuracy of deep learning models in predicting oral cancer survival rates was significantly better than that of decision trees and Cox proportional hazards regression models. ${ }^{24}$

Segmentation and detection are similar tasks in AI, but segmentation mainly aims to define the contours of an object, whereas detection aims to define the position of objects. ${ }^{25}$ Region-based CNN (R-CNN) is commonly utilized in object detection, and its pipelines include extracting region proposals, computing $\mathrm{CNN}$ features and classifying regions. ${ }^{26}$ The most recent algorithm mainly improves proposed region localization efficiency, such as You Only Look Once (YOLO). ${ }^{27}$ Moreover, it was discovered that a deep learning network based on YOLO was superior to oral surgeons in detecting odontogenic cysts in diagnostic function. ${ }^{28}$ Object segmentation required in dentistry is based on pixel-wise segmentation and categorizes each pixel in the region of interest (ROI). Fully convolutional network (FCN) was designed to classify all the pixels using a forward propagation. ${ }^{29}$ In dentistry, delineation is a typical segmentation task that has been applied in tumor diagnosis and radiotherapy planning. The latest Mask R-CNN framework combines object detection and segmentation process, achieving tooth semantic segmentation. ${ }^{30}$ In Mask $\mathrm{R}-\mathrm{CNN}$, ROI align replaces ROI pooling to avoid misalignment, and adds FCN to segment objects in ROI. ${ }^{31}$

\section{Al in orthodontics}

Several reports have indicated that ML has the potential to provide high-quality diagnosis and plan decisionmaking and treatment in the orthodontic field. Compared to traditional procedures, AI simplifies complex protocols, saves time and provides objective predictable outcomes. The 4 major AI-driven tasks can also be applied to orthodontic treatment. Classification tasks are mainly applied to diagnose skeletal type and predict bone age. Regression tasks are applied to cope with clinical decision-making, such as whether or not to extract teeth. Automatic identification of landmarks belongs to detection tasks, and the acquisition of tooth segmentation belongs to segmentation tasks. This section reviews the current progress of ML in orthodontic diagnosis, decision-making, and treatment, and the specific roles in clinical procedures are vividly represented in Fig. 1.

\section{Al-based malocclusion diagnosis}

\section{The automatic identification of landmarks}

The AI-based diagnostic methods significantly reduce technical sensitivity and improve detection accuracy. The supervised learning model is commonly considered the optimal choice for developing an AI-based cephalometric system, in which training data consist of 2 parts: original images and labels. The original image is a lateral cephalogram or CBCT, and their corresponding labels are the desired $\mathrm{X}-\mathrm{Y}$ coordinates of landmarks plotted by orthodontists.

Cheng et al. first applied a model based on random forest to detect the odontoid of the epistropheus, which acts as a baseline of the midsagittal plane. ${ }^{32}$ Although the mean detection error of the model decreased to $3.15 \mathrm{~mm}$, random forest needs to confine the clustered landmarks in the bounding box to avoid searching the whole images. Shahidi et al. utilized feature-based and voxel similaritybased image registration methods to detect 14 landmarks and obtained $<3 \mathrm{~mm}$ mean absolute error (MAE) for $63.57 \%$ of landmarks. ${ }^{33}$ However, to achieve more accurate registration, severe skeletal deformity and fracture were excluded in the trials. Additionally, Montufar et al. used an active shape model combined with knowledgebased local landmark search to predict 18 landmarks and achieved a higher accuracy with an MAE of $2.51 \mathrm{~mm} .^{34}$ 


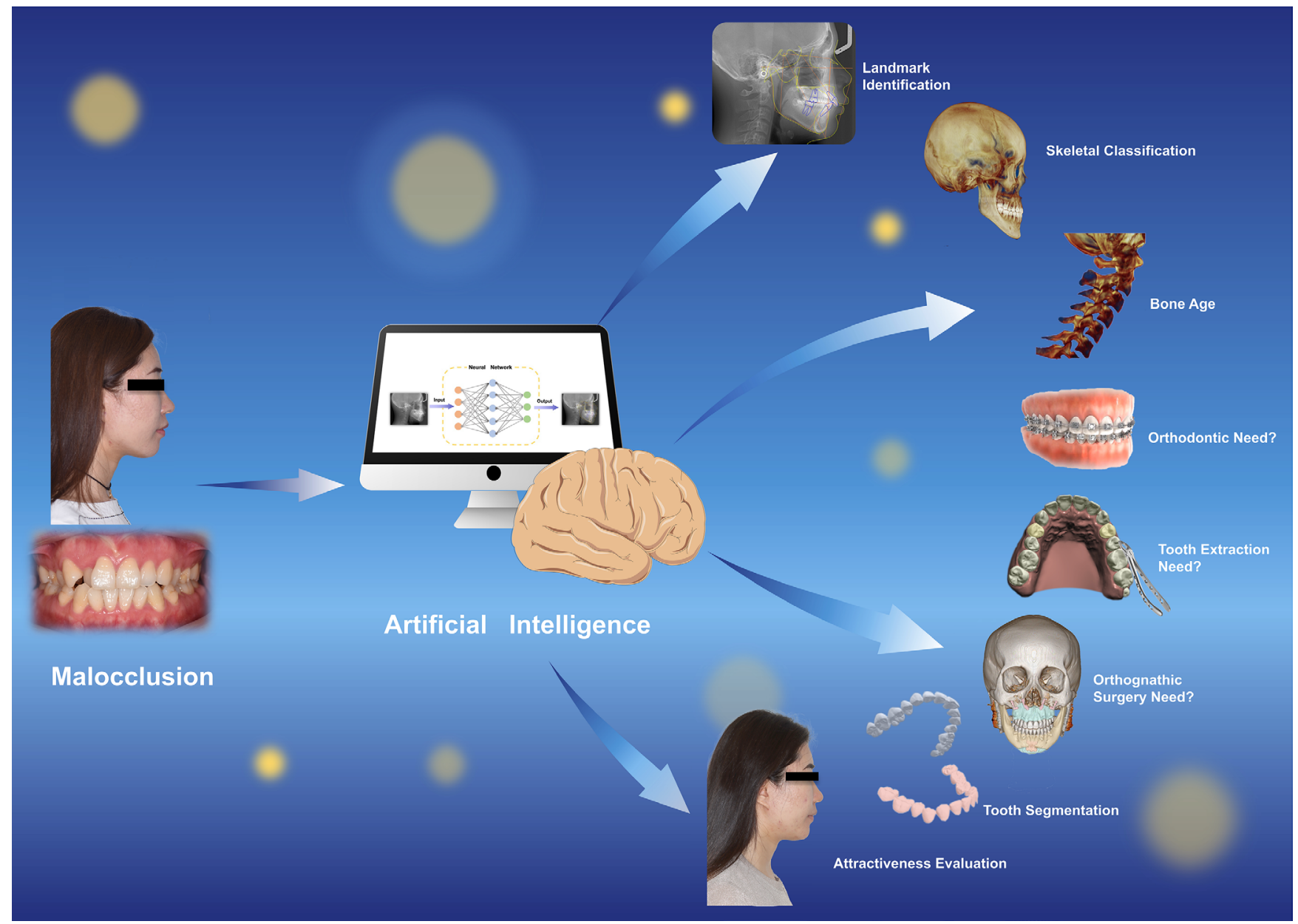

Fig. 1. Schematic representation of the application of artificial intelligence (Al) in orthodontic diagnosis, decision-making and treatment

Nevertheless, these methods rely on significant prior knowledge and still require some manual processes. Furthermore, the methods mentioned above cannot apply to test all individuals due to image size, quality and anatomical variations. Moreover, the handcrafted featurebased ML methods depend on specific algorithm templates for different types of malocclusions.

Compared to traditional ML models, CNN-based models became a desirable solution in actual clinical cases. Japanese scholars first applied the CNN model to identify 10 landmarks using 153 lateral cephalograms for model training. However, the precision was restricted by the limited sample volume and measurement bias. ${ }^{35}$ Kunz et al. expanded the sample to 1792 different cephalograms for training plotted by 12 examiners who were verified by intra-rater and inter-rater reliability calibration. ${ }^{36}$ The MAE of 11 angles and distances calculated using these coordinates predicted by the $\mathrm{CNN}$ model demonstrated no statistically significant differences except for incisor inclination. To improve detection efficiency, an advanced YOLO model, called YOLO v. 3 , showed faster detection and higher accuracy in 1028 cephalograms with less than $0.9 \mathrm{~mm}$ in the MAE of coordinates when comparing to human. ${ }^{37}$ Of note, the R-CNN model could detect soft tissue landmarks to provide a reference for evaluating facial profiles. Meanwhile, according to a sensitivity test, the accuracy of the cephalometric system was not hampered by image quality, gender, skeletal classification, or metallic artifacts. However, the detection of the closely-spaced points was a challenge for $\mathrm{CNN}$-based cephalometric systems. The CBCT can provide higher detail resolution than lateral cephalograms. A geodesic map of mandibles was acquired through linear time distance transformation in CBCT. ${ }^{38} \mathrm{~A}$ CNN model could only locate the sparselyspaced points in the geodesic maps and long short-term memory network framework was applied to capture closely spaced distributed landmarks.

\section{Skeletal classification}

Besides occlusion, the positions of the jaw relative to the cranium are also a valuable index in pre-orthodontic examination. Different types of skeletal malposition were categorized according to Angle classification. Based on ANB angle and Wits appraisal, the anteroposterior position relationship was classified into 3 types: class I (normal), class II (protrusion) and class III (retrusion). Traditional skeletal classification depends on the manual 
calculation of linear and angular variables, using craniomaxillary and mandible landmarks. However, mandible positions vary significantly due to occlusion and temporomandibular joint, which causes difficulties in skeletal classification, while craniomaxillary landmarks are relatively stable. The SVM and ANN can predict mandible variables using craniomaxillary variables, and ANN showed higher correlation coefficients than SVM, especially for gnathion (Gn) and menton (Me) points. Remarkably, the independent variables for producing the best prediction outcomes using ANN came from the literature and from SVM selection. ${ }^{39}$ Besides regression tasks, SVM also generates skeletal classifications based on the automatically extracted craniomaxillary variables. Although the method achieved high sensitivity and precision in predicting class II and III, an unsatisfactory outcome was obtained for class I. This was partially attributed to the misclassification of the cases with marginal values. Better outcomes may be obtained by the combination of synthetic variables and literature variables. ${ }^{40}$

Compared to handcraft methods, CNN-based skeletal classification has demonstrated better classification performance in terms of accuracy, sensitivity and specificity. ${ }^{41}$ Instead of utilizing landmarks, the CNN-based method extracts features by first using the located ROI. The CNNbased method can also divide the vertical skeletal class into normal, hyper- and hypodivergent, in addition to the sagittal relationship. Due to superimposition, the classification precision needs further improvement using CBCT images. Moreover, Kim et al. found the CNN-based model with synchronized multi-channels produced ideal outcomes. ${ }^{42}$ Compared to the single-channel model, better classification performance was achieved by the ensemble and synchronized multi-channel algorithm. However, more influential factors, including race and the wide distribution of normal cases, need to be considered.

\section{Al-based decision-making system}

\section{The prediction of bone age}

The choice of proper treatment timing depends on bone age prediction based on cervical vertebrae maturity, which indicates the deviation extent from normal growth. ${ }^{43}$ The prediction methods of bone age depend on low edge concavities and trapezoid taper of vertebral bodies. ${ }^{44}$ The ML-based methods have shown $>90 \%$ sensitivity, specificity and accuracy for vertical and sagittal skeletal maturation diagnosis. ${ }^{41}$ It has been demonstrated that the accuracy of classification models is superior to clustering models in determining cervical vertebral maturation. ${ }^{45}$ Among a series of classifiers (software), the artificial neural network-based classifier acquired the highest weighted $\kappa$ coefficient (0.926), whereas the lowest value was obtained by a naïve Bayes classifier (0.861). ${ }^{20,46}$ Though lacking accuracy, the discrimination between 2 adjacent stages is not essential to clinical application because the bone development peak occurs between the $3^{\text {rd }}$ and $4^{\text {th }}$ periods. ${ }^{47}$ If the deviation of adjacent stages is neglected, the accuracy will reach 90.42\% using the Bayes classifier. ${ }^{46}$

Besides cervical vertebral maturation, bone age can also be measured using the ossification centers of the proximal phalanx, metacarpal bone and distal radius when the patients suffer from cervical vertebrae deformity. Gao et al. utilized U-Net to acquire mask images of hand bone, and VGGNet was used to perform image classification tasks. ${ }^{48}$ The background noise was removed with hand bone segmentation. Of note, the attention module was inserted into the VGGNet to focus on targeted regions and achieved an MAE of 9.997 months. Although the CNN model with attention modules increases accuracy and effectiveness, attention modules would increase complexity and decrease accuracy in the deeper neural networks, such as ResNet and DenseNet. ${ }^{49}$ Also, due to the additional radiated exposure, manual bone age radiography has been replaced by cervical vertebral maturation assessment using only lateral cephalograms. Therefore, a cervical vertebral maturation degree prediction system based on deep learning requires further research in order to be applied in orthodontic clinical treatment. ${ }^{50}$

\section{The decision on the need for orthodontic treatment}

Traditionally, the decision about whether a patient with malocclusion needs orthodontic treatment is evaluated based on the Index of Orthodontic Treatment Need (ITON) and Dental Aesthetic Index (DAI). ${ }^{51}$ The $\kappa$ values between DAI and ITON in the range of 0.41-0.55 mean that a decision of a specialist is required..$^{51}$ Tedious and complicated tasks can be replaced by decision-making support systems based on ML. Thanathornwong utilized a Bayesian network to look for underlying connections among 15 features of orthodontic patients and obtained conditional probability distributions. ${ }^{52}$ When users input the scores for every index, a probability value would be output, determining whether the patients need orthodontic treatment. The results obtained using the semiautomatic system showed high consistency with 2 orthodontists. Nevertheless, manual interpretation of lateral cephalograms intraoral images may create measurement bias. Besides, the decision system should cover patients of a broader age range, especially the mixed dentition period. Estimating the size of the unerupted teeth plays a vital role in judging whether the patients need early intervention treatment during the mixed dentition period. The prediction primarily depends on Moyer's regression and Tanaka and Johnston's analysis, but the predicting outcomes are not accurate. Moghimi et al. utilized a hybrid genetic algorithm, (GA)-ANN algorithm, to select reference teeth and find the best mapping function to predict the unerupted teeth sizes. ${ }^{53} \mathrm{~A}$ higher proportion of predicting error produced using the GA-ANN algorithm was 
concentrated at $0 \mathrm{~mm}$ and $1 \mathrm{~mm}$, which is acceptable for clinical needs. However, compared to traditional methods used in the treatment of children regardless of different regions and races, future research should improve the generalization capacity of decision systems through high-quality training datasets.

\section{The decision on the need for advance extraction}

The controversy over extraction and non-extraction orthodontic treatment remains unsolved. Currently, orthodontic extraction treatment is applied in cases of dental protrusion and crowding and jaw dysplasia. ${ }^{54}$ Xie et al. described ANN-based decision-making models for determining whether the extraction was necessary. ${ }^{55}$ Although ANN achieved a $100 \%$ success rate in training set using backpropagation, the test outcomes remained at only $80 \%$. Jung et al. chose $1 / 3$ of the training set as a validation set to avoid the overfitting phenomenon. ${ }^{56}$ The proposed method improved the success rate of ANN and achieved an agreement of $94 \%$ with an experienced orthodontist. Additionally, an ANN model consisting of 4 classifiers could not only diagnose the necessity of extraction but also choose proper extraction patterns based on 12 cephalometric variables. Although the accuracy was not ideal in terms of specific extraction positions, only 4 cases were unacceptable for clinical practice. Besides ANN, as one of the ensemble methods, random forest can also prevent overfitting, and random forest showed a lower error rate than ANN regarding the need for extraction and the specific patterns. ${ }^{57}$ Moreover, bagging or boosting can decrease the error rate of the neural network to prevent overfitting. Interestingly, AI-based models discovered that the primary decisive factors for whether to extract teeth before orthodontic treatment are incompetent lips and lower incisor inclination, which can be used as clinical extraction instructions. ${ }^{55}$ However, AI models were used only to predict whether extractions are necessary based on cephalometric outcomes and other measurements. Medical imaging can provide more information than manual measurements and improve the accuracy of diagnosis. Thus, studies of AI-based decision-making models should aim to analyze imaging data. For example, Qin et al. proposed a deep learning method of combining fine-grained features from positron emission tomography (PET) with CT images to diagnose lung cancer noninvasively. ${ }^{58}$ Based on this idea, orthodontists could develop deep learning models for more accurate diagnosis.

\section{The decision on the need for orthognathic surgery}

Due to the higher risk of orthognathic surgery, more comprehensive factors must be taken into consideration, including skeletal classification, facial asymmetry and a patient's chief complaints; all of the above issues bring challenges to clinicians. Therefore, a variety of measurements need to be adopted in the training set to make appropriate decisions. The commonly agreed standard for orthognathic surgery is skeletal class III and asymmetry. Therefore, Knoops et al. utilized an SVM model to diagnose whether patients needed orthognathic surgery using facial scanning images. ${ }^{59}$ Although the system achieved an accuracy of $95.4 \%$, sample selection bias was used because of the fact that normal dentofacial individuals were chosen as non-surgery patients. Jeong et al. chose 822 facial photographs of patients with dentofacial dysmorphosis and/or malocclusion as training data and adopted 3 measurements: facial asymmetry, protrusion and retrusion. ${ }^{60}$ The CNN model could extract the profile feature from the front and side facial photographs, and subsequently divided the cases into surgery and non-surgery. To integrate more factors, Choi et al. incorporated more measures such as E-line and occlusal plane into ML models, but the feature values required manual input instead of automatic image extraction. ${ }^{61}$ The ANN model provided high accuracy during the test stage without overfitting. Besides deciding whether to operate or not, the system can also predict the demand for tooth extraction for surgical patients using 4 classifiers. Obtaining right answers is not the purpose of AI-based models because excessive feature information increases the algorithm complexity and overfitting risk. More importantly, the decision-making system can provide diverse clinical solutions for inexperienced orthodontists.

\section{Al-based orthodontic treatment}

\section{The acquisition of tooth segmentation images}

Template-based registration has been applied to segment target teeth contours, but the application of the method is limited by image intensity and anatomic similarity. ${ }^{62}$ Conversely, the level set method can handle the images with anatomic variation, low resolution and noise, but it performs poorly in delineating the edge contours, especially in the interdental areas. ${ }^{63} \mathrm{Kim}$ et al. removed the interdental area with masks and reconstructed the edge contours using Generative Adversarial Networks (GAN) ${ }^{64}$ Compared to separated scanning without interdental areas, the proposed method improved the precision to $0.004 \mathrm{~mm}$. However, the size of the mask was inversely associated with the accuracy of the reconstruction, due to the masking of adjacent normal structures. Pose regression was found to eliminate the overlapping areas by realigning the volume-of-interest regions. ${ }^{26}$ Moreover, it was discovered that for tooth segmentation, the pose-aware R-CNN model outperformed the Mask R-CNN model in accuracy and sensitivity. ${ }^{26,65}$ Due to redundant region proposals in $\mathrm{R}-\mathrm{CNN}$ algorithms, similarity matrix and non-maximum suppression can both mine the region proposal network. Cui et al. found similarity matrix to show superiority to non-maximum suppression in segmentation 
accuracy. ${ }^{65}$ Additionally, tooth segmentation may often be interfered with by extreme gray values, such as metal artifacts. Chung et al. reported the cutout augmentation method to achieve metal artifacts segmentation. ${ }^{26}$ Additionally, the edge map combined with original CBCT images enhanced boundary information and caused accuracy improvement. ${ }^{65} \mathrm{With}$ integrity of the spatial relation and shape features, Mask R-CNN was able to achieve tooth instance segmentation. ${ }^{65}$

\section{The evaluation of treatment effect}

Improving facial attractiveness is one of the main reasons for choosing orthodontic treatment. Therefore, it is important for orthodontists to provide patients with an objective and esthetical assessment. Although attractiveness is based in cognitive psychology, ML can act as a useful tool to reflect professional and social esthetic appreciation. The CNN model can simulate humans in grading the attractiveness of facial photographs utilizing facial detection and feature extraction. The outcomes produced by the CNN model were more closely aligned with orthodontists and oral surgeons than laypersons. Meanwhile, the variation resulted in fewer of the same targets. Therefore, these results reflected superior reliability of the CNNbased facial evaluation system. However, the CNN model could not explain the influence of each facial feature on facial attractiveness. ${ }^{66}$ Zhao et al.utilized ML algorithms to predict facial attractiveness when considering different features, including facial shape, geometric features and triangle area features. ${ }^{67}$ Importantly, facial shape played a significant role in determining facial attractiveness. The treatment effect differed among different patients due to the treatment planning and individual conditions of a patient, including hairstyle and facial ratio. Moreover,
ML can help to evaluate the influence of plastic treatment on patients' attractiveness. Currently, some scholars found that orthognathic surgery improved facial attractiveness and decreased estimated age by 1.2 years. Significant improvement of facial attractiveness could be seen in patients with facial asymmetry and skeletal class II and III. Additionally, lower jaw osteotomy could most clearly ameliorate facial attractiveness. ${ }^{68}$ What is important, facial esthetics are influenced by a variety of factors, so we need to reduce the influence of confounding factors such as skin, hairstyle and lighting conditions. Also, as training data, esthetic review bias needs further validation, so global standardized facial databases are recommended.

\section{Limitations and perspectives}

The rapid development of AI have contributed to its promising applications in dentistry. Accordingly, a variety of AI-based products have been developed by several companies to assist dental clinical applications such as radiological diagnosis and decision-making, as summarized in Table 1. Due to the advantages of high-dimensional data mining, deep learning models have shown great potential in dentistry, especially in oral oncology. Recently, multifeature concatenation method has been applied to diagnose cervical lymph node metastasis, enhancing target detection efficiency. ${ }^{69}$ Additionally, deep convolutional GAN has become an effective solution to predict reconstructive jaw morphology before surgeries, which facilitates accurate 3D printing. ${ }^{70}$ Breakthroughs in other dental fields can shed light on the application of AI in orthodontics. Despite its potential, many challenges, such as data insufficiency, reproducibility crisis and overfitting need to be solved before AI is implemented into clinical practice.

Table 1. The companies and their representative products based on machine learning $(\mathrm{ML})$ in dental field

\begin{tabular}{|c|c|c|c|c|}
\hline Company & Product & Function & Website & Origin \\
\hline Denti.Al & Denti.Al & dental disease detection & https://www.denti.ai/ & Toronto, Canada \\
\hline VideaHealth & VideaHealth & dental disease detection & https://www.videa.ai/ & Boston, USA \\
\hline Pearl & Second Opinion & dental disease detection & https://www.hellopearl.com/ & West Hollywood, USA \\
\hline Dentem & Dx Vision & dental problem detection & https://www.dentem.co/ & Toronto, Canada \\
\hline Medihome & Medihome & dental disease detection & http://medihome.jp/ & Tokyo, Japan \\
\hline DeepCare & $\begin{array}{l}\text { DeepCare Dental } \\
\text { Landscape }\end{array}$ & dental disease detection & http://www.deepcare.com/ & Beijing, China \\
\hline ORCA Dental Al & Cephx & $\begin{array}{l}\text { cephalometric analysis } \\
\text { teeth segmentation } \\
\text { airway analysis }\end{array}$ & https://www.orca-ai.com/ & Las Vegas, USA \\
\hline CellmatiQ GmbH & $\begin{array}{l}\text { DentaliQ ortho } \\
\text { DentaliQ opg }\end{array}$ & $\begin{array}{l}\text { cephalometric analysis } \\
\text { dental disease detection }\end{array}$ & https://cellmatiq.com/ & Hamburg, Germany \\
\hline Uceph & Uceph & cephalometric analysis & http://www.uceph.com/ & Chengdu, China \\
\hline Zhibeicloud & Zhibeicloud & $\begin{array}{l}\text { cephalometric analysis } \\
\text { bone age analysis } \\
\text { facial esthetic analysis }\end{array}$ & https://www.aortho360.com/ & Chengdu, China \\
\hline
\end{tabular}




\section{Data insufficiency}

Compared to other medical fields, the high-quality datasets for orthodontic research are limited. The discrepancies in training data make the comparison of different AI-based models questionable. Although supervised learning is currently the optimal choice in malocclusion diagnosis, huge costs and labor required for target labeling create obstacles in creating standardized and high-quality datasets for orthodontic studies, specifically. Additionally, data snooping bias usually occurs because training data are repeatedly applied in the test stage. Therefore, independent datasets for tests should be applied instead of cross-validation. To solve this problem, semi-supervised and weakly supervised learning framework can be used in analyzing many original images, and show comparable accuracy and robustness in diagnosis with supervised learning. ${ }^{71}$ Moreover, transfer learning and few-shot learning could also become ideal alternatives to solve data insufficiency problems. ${ }^{41}$

\section{Reproducibility crisis}

An increasing number of scientists have realized the reproducibility crisis of AI, which means that many research results cannot be repeated when the same experiment is conducted by another team of scientists. The reasons for this phenomenon include algorithmic and metric knowledge deficiency, as well as misunderstanding. Additionally, many researchers neglect the sensitivity of results to hyper-parameters, including study rate, iteration times and initialization strategy. There are some effective solutions to improve the trustworthiness of AI. For instance, sensitivity tests need to be carried out when evaluating model performance. Furthermore, the improvement of interpretability obtained through visualizing the mechanisms of ML models could aid in solving the dilemma. Of note, data cleaning method can be an effective alternative to prevent manual errors in labeling and measurement from affecting the reproducibility of ML models.

\section{Overfitting problem}

Most feature engineering models are confronted with the overfitting problem, i.e., worse performance of the models in predicting unknown samples. Many reasons account for overfitting as, for example, data for testing and training are normally derived from a common internal dataset. Additionally, health data heterogeneity is a key factor. Several improvements can be made to prevent overfitting. Firstly, to improve the external generalization capacities of ML models, greater and more diverse external clinical scenarios are required to validate the performance of the model. Meanwhile, choosing representative datasets needs to be considered. Furthermore, several algorithms have been created to prevent overfitting, such as early stopping, dropout and regularization penalty. ${ }^{72}$ Multimodal learning can integrate various types of information, including records and images, and reach ideal performance when processing imbalanced data. ${ }^{73}$

\section{The next step}

Besides improving the experimental performance of $\mathrm{ML}$, the question of how to apply AI to revolutionize traditional orthodontic procedures should be considered at present. First of all, due to the black-box feature of ML, enhancing the visualization and establishing patients' and doctors' trust should be prioritized before clinical application of ML. Although the interpretability of ML still remains challenging, standardized clinical trials should be conducted, which can serve as strong medical evidence for guidance. During trial design, several methods are needed to control bias risk. For instance, it is essential to conduct inter-rater reliability calibration using consistency validation. Additionally, the allocation schemes should be blind to reviewers to prevent subjective bias. Moreover, providing context-specific empirical validation could also make it easier to audit bias and enhance trust of practitioners, instead of explaining inner workflows.

In the next few years, AI-based orthodontic systems could serve as an auxiliary tool for clinical procedures. The outcomes derived from ML models should be treated cautiously and used as a reference. Additionally, the AIbased models can simulate experts' ways of thinking and provide the advice for orthodontists. Through ML models, acquisition of theories and practice experiences by young orthodontists could become easier and faster. In addition, with the help of nature language process, ML models could evaluate evidence quality by extracting information from published papers. The evidence evaluation system could relieve residents' pressure in acquiring medical evidence.

The ethical problems in implementing ML in orthodontic procedures cannot be neglected. Firstly, it is the inherent right of subjects to know about the principles, functions and limitations of the ML models. Secondly, the doctor's sense of responsibility should be emphasized when establishing cooperation with patients. Thirdly, health data privacy should be redefined due to the prevalence of electronic medical records. Lastly, the legal liability distribution between doctors and ML models should be further improved.

\section{Conclusions}

With the advancement of AI in dentistry, high-quality disease diagnosis, decision-making and treatment can be achieved in the near future. However, there are many studies relating to the image recognition, while decision support systems have received little attention. Additionally, clinicians should view AI as a support for diagnosis and 
treatment, not as a threat. Moreover, clinicians need to be cautious about the prediction outcomes provided by AI models before the interpretability is fully clarified. Due to the growing emphasis on medical responsibility and ethical principles, legal recognition of $\mathrm{AI}$ is also a crucial issue. In the future, more clinical trials regarding the application of AI in orthodontics should be carried out to revolutionize traditional orthodontic treatment procedures.

\section{ORCID iDs}

Jialing Liu (ib https://orcid.org/0000-0001-5554-0870 Ye Chen (1) https://orcid.org/0000-0002-7217-366X Shihao Li (1) https://orcid.org/0000-0002-6418-7818 Zhihe Zhao (1) https://orcid.org/0000-0003-2955-1706 Zhihong Wu (1) https://orcid.org/0000-0001-5712-2285

\section{References}

1. Lombardo G, Vena F, Negri P, et al. Worldwide prevalence of malocclusion in the different stages of dentition: A systematic review and meta-analysis. Eur J Paediatr Dent. 2020;21(2):115-122. doi:10.23804/ ejpd.2020.21.02.05

2. Gribel BF, Gribel MN, Frazao DC, McNamara JA Jr, Manzi FR. Accuracy and reliability of craniometric measurements on lateral cephalometry and 3D measurements on CBCT scans. Angle Orthod. 2011;81(1): 26-35. doi:10.2319/032210-166.1

3. Konstantonis D, Vasileiou D, Papageorgiou SN, Eliades T. Soft tis sue changes following extraction vs. nonextraction orthodontic fixed appliance treatment: A systematic review and meta-analysis. Eur J Oral Sci. 2018;126(3):167-179. doi:10.1111/eos.12409

4. da Pozzo F, Gibelli D, Beltramini GA, Dolci C, Gianni AB, Sforza C. The effect of orthognathic surgery on soft-tissue facial asymmetry: A longitudinal three-dimensional analysis. J Craniofac Surg. 2020. 31(6):1578-1582. doi:10.1097/SCS.0000000000006403

5. Papadimitriou A, Mousoulea S, Gkantidis N, Kloukos D. Clinical effectiveness of Invisalign(R) orthodontic treatment: A systematic review. Prog Orthod. 2018;19(1):37. doi:10.1186/s40510-018-0235-Z

6. Gan Y, Xia Z, Xiong J, Li G, Zhao Q. Tooth and alveolar bone segmentation from dental computed tomography images. IEEE J Biomed Health Inform. 2018;22(1):196-204. doi:10.1109/JBHI.2017.2709406

7. Yuan T, Wang Y, Hou Z, Wang J. Tooth segmentation and gingival tissue deformation framework for 3D orthodontic treatment planning and evaluating. Med Biol Eng Comput. 2020;58(10):2271-2290. doi:10.1007/s11517-020-02230-9

8. Machoy ME, Szyszka-Sommerfeld L, Vegh A, Gedrange T, Woźniak K. The ways of using machine learning in dentistry. Adv Clin Exp Med. 2020;29(3):375-384. doi:10.17219/acem/115083

9. Waldron T, Carr T, McMullen L, et al. Development of a program theory for shared decision-making: A realist synthesis. BMC Health Serv Res. 2020;20(1):59. doi:10.1186/s12913-019-4649-1

10. Schwendicke F, Samek W, Krois J. Artificial intelligence in dentistry: Chances and challenges. J Dent Res. 2020;99(7):769-774. doi:10.1177/ 0022034520915714

11. Chen D, Wang Z, Guo D, Orekhov V, Qu X. Review and prospect: Deep learning in nuclear magnetic resonance spectroscopy. Chemistry. 2020;26(46):10391-10401. doi:10.1002/chem.202000246

12. Yoon $\mathrm{HJ}, \mathrm{Kim} \mathrm{S}, \mathrm{Kim} \mathrm{JH}$, et al. A lesion-based convolutional neural network improves endoscopic detection and depth prediction of early gastric cancer. J Clin Med. 2019;8(9):1310. doi:10.3390/jcm8091310

13. Wang L, Yang Y, Min R, Chakradhar S. Accelerating deep neural network training with inconsistent stochastic gradient descent. Neural Netw. 2017;93:219-229. doi:10.1016/j.neunet.2017.06.003

14. Hu X, Yi W, Jiang $L$, et al. Classification of metaphase chromosomes using deep convolutional neural network. J Comput Biol. 2019;26(5): 473-484. doi:10.1089/cmb.2018.0212

15. Luo X, Chi W, Deng M. Deepprune: Learning efficient and interpretable convolutional networks through weight pruning for predicting DNA-protein binding. Front Genet. 2019;10:1145. doi:10.3389/ fgene.2019.01145
16. Mellouli D, Hamdani TM, Sanchez-Medina JJ, Ben Ayed M, Alimi AM. Morphological convolutional neural network architecture for digit recognition. IEEE Trans Neural Netw Learn Syst. 2019;30(9):2876-2885. doi:10.1109/TNNLS.2018.2890334

17. Ilyas N, Shahzad A, Kim K. Convolutional-neural network-based image crowd counting: Review, categorization, analysis, and performance evaluation. Sensors (Basel). 2019;20(1):43. doi:10.3390/s20010043

18. Holder LB, Haque MM, Skinner MK. Machine learning for epigenetics and future medical applications. Epigenetics. 2017;12(7):505-514. doi:10.1080/15592294.2017.1329068

19. Zhang $Y$, Lin $H$, Yang Z, et al. Neural network-based approaches for biomedical relation classification: A review. J Biomed Inform. 2019;99: 103294. doi:10.1016/j.jbi.2019.103294

20. Amasya H, Yildirim D, Aydogan T, Kemaloglu N, Orhan K. Cervical vertebral maturation assessment on lateral cephalometric radiographs using artificial intelligence: Comparison of machine learning classifier models. Dentomaxillofac Radiol. 2020;49(5):20190441. doi:10.1259/ dmfr.20190441

21. Wang EK, Xi L, Sun RP, et al. A new deep learning model for assisted diagnosis on electrocardiogram. Math Biosci Eng. 2019;16(4):2481-2491. doi:10.3934/mbe.2019124

22. Das N, Hussain E, Mahanta LB. Automated classification of cells into multiple classes in epithelial tissue of oral squamous cell carcinoma using transfer learning and convolutional neural network. Neural Netw. 2020;128:47-60. doi:10.1016/j.neunet.2020.05.003

23. Kawahara A, Sato T, Hayashi K. Multivariate regression analysis to predict postoperative refractive astigmatism in cataract surgery. J Ophthalmol. 2020;2020:9842803. doi:10.1155/2020/9842803

24. Kim DW, Lee S, Kwon S, Nam W, Cha IH, Kim HJ. Deep learning-based survival prediction of oral cancer patients. Sci Rep. 2019;9(1):6994. doi:10.1038/s41598-019-43372-7

25. Rajan PG, Sundar C. Brain tumor detection and segmentation by intensity adjustment. J Med Syst. 2019;43(8):282. doi:10.1007/s10916019-1368-4

26. Chung $M$, Lee $M$, Hong J, et al. Pose-aware instance segmentation framework from cone-beam CT images for tooth segmentation. Comput Biol Med.2020;120:103720. doi:10.1016/j.compbiomed.2020. 103720

27. Liu JL, Li SH, Cai YM, et al. Automated radiographic evaluation of adenoid hypertrophy based on VGG-Lite. J Dent Res. 2021;2021:220 345211009474. doi:10.1177/00220345211009474

28. Yang $\mathrm{H}$, Jo $\mathrm{E}, \mathrm{Kim} \mathrm{HJ}$, et al. Deep learning for automated detection of cyst and tumors of the jaw in panoramic radiographs. J Clin Med. 2020;9(6):1839. doi:10.3390/jcm9061839

29. Li H, Jiang G, Zhang J, et al. Fully convolutional network ensembles for white matter hyperintensities segmentation in MR images. Neuroimage. 2018;183:650-665. doi:10.1016/j.neuroimage.2018.07.005

30. Zhang L, Wu J, Fan Y, Gao H, Shao Y. An efficient building extraction method from high spatial resolution remote sensing images based on improved mask R-CNN. Sensors (Basel). 2020;20(5):1465. doi:10.3390/s20051465

31. Zhang Y, Chu J, Leng L, Miao J. Mask-refined R-CNN: A network for refining object details in instance segmentation. Sensors (Basel). 2020;20(4):1010. doi:10.3390/s20041010

32. Cheng E, Chen J, Yang J, et al. Automatic dent-landmark detection in 3-D CBCT dental volumes. Annu Int Conf IEEE Eng Med Biol Soc. 2011;2011:6204-6207. doi:10.1109/IEMBS.2011.6091532

33. Shahidi S, Bahrampour E, Soltanimehr E, et al. The accuracy of a designed software for automated localization of craniofacial landmarks on CBCT images. BMC Med Imaging. 2014;14:32. doi:10.1186/ 1471-2342-14-32

34. Montufar J, Romero M, Scougall-Vilchis RJ. Hybrid approach for automatic cephalometric landmark annotation on cone-beam computed tomography volumes. Am J Orthodont Dentofacial Orthop. 2018; 154(1):140-150. doi:10.1016/j.ajodo.2017.08.028

35. Nishimoto S, Sotsuka Y, Kawai K, Ishise H, Kakibuchi M. Personal computer-based cephalometric landmark detection with deep learning, using cephalograms on the Internet. J Craniofacial Surg. 2019; 30(1):91-95. doi:10.1097/scs.0000000000004901

36. Kunz F, Stellzig-Eisenhauer A, Zeman F, Boldt J. Artificial intelligence in orthodontics. J Orofacial Orthoped. 2019;81(1):52-68. doi:10.1007/ s00056-019-00203-8 
37. Hwang HW, Park JH, Moon JH, et al. Automated identification of cephalometric landmarks. Part 2: Might it be better than human? Angle Orthod. 2020;90(1):69-76. doi:10.2319/022019-129.1

38. Torosdagli N, Liberton DK, Verma P, Sincan M, Lee JS, Bagci U. Deep geodesic learning for segmentation and anatomical landmarking. IEEE Trans Med Imaging. 2019;38(4):919-931. doi:10.1109/tmi.2018. 2875814

39. Nino-Sandoval TC, Guevara Perez SV, Gonzalez FA, Jaque RA, Infante-Contreras $C$. Use of automated learning techniques for predicting mandibular morphology in skeletal class I, II and III. Forensic Sci Int. 2017;281:187.e1-187.e7. doi:10.1016/j.forsciint.2017.10.004

40. Nino-Sandoval TC, Guevara Perez SV, Gonzalez FA, Jaque RA, Infante-Contreras C. An automatic method for skeletal patterns classification using craniomaxillary variables on a Colombian population. Forensic Sci Int. 2016;261:159.e1-e6. doi:10.1016/j.forsciint.2015.12.025

41. Yu HJ, Cho SR, Kim MJ, Kim WH, Kim JW, Choi J. Automated skeletal classification with lateral cephalometry based on artificial intelligence. J Dent Res. 2020;99(3):249-256. doi:10.1177/0022034520901715

42. Kim I, Misra D, Rodriguez L, et al. Malocclusion classification on 3D cone-beam CT craniofacial images using multi-channel deep learning models. Annu Int Conf IEEE Eng Med Biol Soc. 2020;2020:1294-1298. doi:10.1109/EMBC44109.2020.9176672

43. Durka-Zajac M, Mitus-Kenig M, Derwich M, Marcinkowska-Mitus A, Loboda M. Radiological indicators of bone age assessment in cephalometric images: Review. Pol J Radiol. 2016;81:347-353. doi:10.12659/ PJR.895921

44. McNamara JA Jr, Franchi L. The cervical vertebral maturation method: A user's guide. Angle Orthod. 2018;88(2):133-143. doi:10.2319/111517787.1

45. Sokic E, Tiro A, Sokic-Begovic E, Nakas E, Tiro A. Semi-automatic assessment of cervical vertebral maturation stages using cephalograph images and centroid-based clustering. Acta Stomatol Croat. 2012;46(4):280-290.

46. Baptista RS, Quaglio CL, Mourad LMEH, et al. A semi-automated method for bone age assessment using cervical vertebral maturation. Angle Orthod. 2012;82(4):658-662. doi:10.2319/070111-425.1

47. Gu Y, McNamara JA. Mandibular growth changes and cervical vertebral maturation: A cephalometric implant study. Angle Orthod. 2007;77(6):947-953. doi:10.2319/071006-284.1

48. Gao Y, Zhu T, Xu X. Bone age assessment based on deep convolution neural network incorporated with segmentation. Int J Comput Assist Radiol Surg. 2020;15(12):1951-1962. doi:10.1007/s11548-020-02266-0

49. Tajmir SH, Lee H, Shailam R, et al. Artificial intelligence-assisted interpretation of bone age radiographs improves accuracy and decreases variability. Skeletal Radiol. 2019;48(2):275-283. doi:10.1007/s00256018-3033-2

50. Makaremi M, Lacaule C, Mohammad-Djafari A. Deep learning and artificial intelligence for the determination of the cervical vertebra maturation degree from lateral radiography. Entropy. 2019;21(12). doi:10.3390/e21121222

51. Vedovello SAS, Dos Santos PR, Mello de Carvalho AL, et al. Exploring the perception of orthodontic treatment need using the Dental Aesthetic Index and Index of Orthodontic Treatment Need. Am J Orthod Dentofacial Orthop. 2019;156(6):818-822. doi:10.1016/j. ajodo.2019.01.020

52. Thanathornwong B. Bayesian-based decision support system for assessing the needs for orthodontic treatment. Healthc Inform Res. 2018;24(1):22-28. doi:10.4258/hir.2018.24.1.22

53. Moghimi S, Talebi M, Parisay I. Design and implementation of a hybrid genetic algorithm and artificial neural network system for predicting the sizes of unerupted canines and premolars. Eur J Orthod. 2011; 34(4):480-486. doi:10.1093/ejo/cjr042

54. Soheilifar $\mathrm{S}$, Soheilifar $\mathrm{S}$, Ataei $\mathrm{H}$, et al. Extraction versus non-extraction orthodontic treatment: Soft tissue profile changes in borderline class I patients. Dent Med Probl. 2020;57(3):275-283. doi:10.17219/ dmp/119102
55. XieX, Wang L, Wang A. Artificial neural network modeling for deciding if extractions are necessary prior to orthodontic treatment. Angle Orthod. 2010;80(2):262-266. doi:10.2319/111608-588.1

56. Jung SK, Kim TW. New approach for the diagnosis of extractions with neural network machine learning. Am J Orthod Dentofacial Orthop. 2016;149(1):127-133. doi:10.1016/j.ajodo.2015.07.030

57. Suhail Y, Upadhyay M, Chhibber A, Kshitiz J. Machine learning for the diagnosis of orthodontic extractions: A computational analysis using ensemble learning. Bioengineering (Basel). 2020;7(2):55. doi:10.3390/bioengineering7020055

58. Qin RX, Wang ZZ, Jiang LY, et al. Fine-grained lung cancer classification from PET and CT images based on multidimensional attention mechanism. Complexity. 2020;12:6153657. doi:10.1155/2020/6153657

59. Knoops PGM, Papaioannou A, Borghi A, et al. A machine learning framework for automated diagnosis and computer-assisted planning in plastic and reconstructive surgery. Sci Rep. 2019;9(1):13597. doi:10.1038/s41598-019-49506-1

60. Jeong SH, Yun JP, Yeom HG, Lim HJ, Lee J, Kim BC. Deep learning based discrimination of soft tissue profiles requiring orthognathic surgery by facial photographs. Sci Rep. 2020;10(1):16235. doi:10.1038/ s41598-020-73287-7

61. Choi HI, Jung SK, Baek SH, et al. Artificial intelligent model with neural network machine learning for the diagnosis of orthognathic surgery. J Craniofac Surg. 2019;30(7):1986-1989. doi:10.1097/SCS.00000 00000005650

62. Barone S, Paoli A, Razionale AV. CT segmentation of dental shapes by anatomy-driven reformation imaging and B-spline modelling. Int J Numer Method Biomed Eng. 2016;32(6). doi:10.1002/cnm.2747

63. Gan Y, Xia Z, Xiong J, Zhao Q, Hu Y, Zhang J. Toward accurate tooth segmentation from computed tomography images using a hybrid level set model. Med Phys. 2014;42(1):14-27. doi:10.1118/1.4901521

64. Kim T, Cho Y, Kim D, Chang M, Kim YJ. Tooth segmentation of 3D scan data using generative adversarial networks. Appl Sci. 2020;10(2):490. doi:10.3390/app10020490

65. Cui Z, Li C, Wang W. ToothNet: Automatic tooth instance segmentation and identification from cone beam CT images. Paper presented at: 2019 IEEE/CVF Conference on Computer Vision and Pattern Recognition (CVPR); June 15-20 2019; Long Beach, USA. doi:10.1109/ CVPR.2019.00653

66. Patcas R, Timofte R, Volokitin A, et al. Facial attractiveness of cleft patients: A direct comparison between artificial-intelligence-based scoring and conventional rater groups. Eur JOrthod. 2019;41(4):428-433. doi:10.1093/ejo/cjz007

67. Zhao J, Zhang M, He C, Xie X, Li J. A novel facial attractiveness evaluation system based on face shape, facial structure features and skin. Cogn Neurodyn. 2020;14(5):643-656. doi:10.1007/s11571-020-09591-9

68. Patcas R, Bernini DAJ, Volokitin A, Agustsson E, Rothe R, Timofte R. Applying artificial intelligence to assess the impact of orthognathic treatment on facial attractiveness and estimated age. Int J Oral Maxillofac Surg. 2019;48(1):77-83. doi:10.1016/j.ijom.2018.07.010

69. Shih KH, Chiu CT, Lin JA, Bu YY. Real-time object detection with reduced region proposal network via multi-feature concatenation. IEEE Trans Neural Netw Learning Syst. 2020;31(6):2164-2173. doi:10.1109/TNNLS. 2019.2929059

70. Liang Y, Huan J, Li JD, Jiang C, Fang C, Liu Y. Use of artificial intelligence to recover mandibular morphology after disease. Sci Rep. 2020;10(1):16431. doi:10.1038/s41598-020-73394-5

71. Han CH, Kim M, Kwak JT. Semi-supervised learning for an improved diagnosis of COVID-19 in CT images. PLoS One. 2021;16(4):e0249450. doi:10.1371/journal.pone.0249450

72. Leite $A F$, Gerven AV, Willems $H$, et al. Artificial intelligence-driven novel tool for tooth detection and segmentation on panoramic radiographs. Clin Oral Investig. 2020;25(4):2257-2267. doi:10.1007/s00784020-03544-6

73. Dai L, Fang $\mathrm{R}$, Li H, et al. Clinical report-guided retinal microaneurysm detection with multi-sieving deep learning. IEEE Trans Med Imaging. 2018;37(5):1149-1161. doi:10.1109/TMI.2018.2794988 